\title{
Early childhood language outcomes after arterial switch operation: a prospective cohort study
}

\author{
Matt S. Hicks', Reginald S. Sauve ${ }^{2,5^{*}}$, Charlene M. T. Robertson ${ }^{1,3}$, Ari R. Joffe' ${ }^{1}$ Gwen Alton ${ }^{1}$, Dianne Creighton², \\ David B. Ross ${ }^{4}$, Ivan M. Rebeyka ${ }^{1,4}$ and for the Western Canadian Complex Pediatric Therapies Follow-up Group
}

\begin{abstract}
Background: Developmental and language outcomes at 2 years of age of children who had arterial switch operation (ASO) for transposition of the great arteries 2004-2010 are described.

Methods: In this prospective cohort study, $91 / 98$ (93 \%) children who underwent ASO were assessed at 2 years of age with the Bayley Scales of Infant \& Toddler Development-3rd Edition. Outcomes were compared by patient and perioperative variables using bivariate and multivariate regression analyses to identify predictors of language delay.

Results: Infants without ventricular septal defect (VSD) $(n=60)$ were more likely to be outborn (73 vs $58 \%$, $p=0.038$ ), require septostomy ( 80 vs $58 \%, p=0.026$ ), have a shorter cross clamp time (min) (62.7 vs 73.0, $p=0.019$ ), and a lower day 1 post-operative plasma lactate $(\mathrm{mmol} / \mathrm{L})(3.9 \mathrm{vs} 4.8, \mathrm{p}=0.010)$. There were no differences in cognitive, motor and language outcomes based on presence of a VSD. Language delay $(<85)$ of $29 \%$ was 1.8 times higher than the normative sample; risk factors for this in multivariate analyses included $<12$ years of maternal education (AOR $19.3,95 \% \mathrm{Cl} 2.5-148.0$ ) and cross-clamp time $\geq 70 \mathrm{~min}$ (AOR 14.5, $95 \% \mathrm{Cl}$ 3.1-68.5). Maternal education $<12$ years was associated with lower Language Composite Scores $(-20.2,95 \% \mathrm{Cl}-32.3$ to -9.1$)$.

Conclusions: Outcomes at 2 years of age in children who undergo ASO are comparable to the normative sample with the exception of language. There is a risk of language delay for which maternal education and cross-clamp duration are predictors. These findings suggest that focused post-operative early language interventions could be considered.
\end{abstract}

Keywords: Transposition of great vessels, Follow-up studies, Child development, Thoracic surgery, Cohort studies

\section{Background}

Arterial switch operation (ASO) is the main surgical modality for uncomplicated transposition of the great arteries (TGA) (Freed et al. 2006). Developmental and language delays have been described in previous cohorts with TGA following ASO, however there has since been evolution of perioperative management strategies that may have led to further improvement in outcomes (Bellinger et al. 1999; Hövels-Gürich et al. 2002; Williams 2003; Freed et al. 2006). Overall long term neurocognitive,

\footnotetext{
*Correspondence: rsauve@ucalgary.ca

${ }^{5}$ Department of Community Health Sciences, Cumming School

of Medicine, University of Calgary, Calgary, AB, Canada

Full list of author information is available at the end of the article
}

health and motor outcomes have improved over time and in most recent studies these are comparable to the general population with the exception of differences in executive function, academic achievement, behaviour and motor skills (Bellinger et al. 1995, 1999; Gaynor et al. 2007). Language delay out of keeping with overall neurocognitive function has been reported in several cohorts following repair of TGA over the last 3 decades (Bellinger et al. 1999; Hövels-Gürich et al. 2002; Bellinger et al. 2003; Neufeld et al. 2008). A report of a cohort from a previous surgical era at our center found increased rates of intellectual disability, autism spectrum disorder and language delay at 5 years of age (Neufeld et al. 2008). Social class, cardio-pulmonary bypass $(\mathrm{CPB})$ duration, clinical and subclinical seizures, and ventricular septal defect (VSD) 
have been identified as predictors of developmental outcome (Bellinger et al. 1995; Rappaport et al. 1998; Hövels-Gürich et al. 2002; Wypij et al. 2003; Beca et al. 2009). In this article the early neurocognitive and language outcomes for children undergoing ASO in a tertiary center in the period 2004-2010 are described and children were stratified by presence or absence of a VSD to determine if this remains a risk factor for poorer outcome.

\section{Methods}

This cohort study enrolled 102 consecutive children who underwent ASO for repair of TGA at Stollery Children's Hospital, Edmonton, Alberta in the period July 2004 to June 2010. This study included all children with an anatomic diagnosis of TGA with or without VSD. Cardiac surgery was performed by two cardiac surgeons (DBR, IMR). Operative procedures included full-flow cardiopulmonary bypass $(\mathrm{CPB})$, moderate hypothermia and deep hypothermic circulatory arrest (DHCA) in $66 \%$ of cases and a minimum hematocrit value of $0.25 \%$. Use of DHCA was generally reserved for the short period required to close the ASD. Venous cannulation strategy was based upon surgeon preference. Intraoperative lactate measurements were not routinely collected. Perioperative electroencephalograms (EEGs) were not obtained unless there was clinical concern of seizure. Near-infrared spectroscopy (NIRS) has recently been introduced to the clinical environment to measure cerebral oxygenation but these data are not available for the entire cohort. Perioperative and demographic characteristics were collected prospectively and included the following: demographics (sex, race, socioeconomic status-Blishen, maternal education, delivery at a hospital outside of the region of the operating site or outborn, and year of operation), birth characteristics (antenatal diagnosis, gestational age, birth weight, use of balloon atrial septostomy (BAS), and age at operation), peri-operative measures of illness severity (modified inotrope score, highest plasma lactate value, total days of ventilation, presence of convulsions, sepsis, renal insufficiency as defined by need for dialysis or creatinine $>100 \mu \mathrm{mol} / \mathrm{L}$ and total hospital days at the operative site), and operative characteristics (CPB time, cross-clamp time, and use of DHCA) (Blishen et al. 1987). The postoperative modified inotrope score was calculated as per Wernovsky and colleagues (Wernovsky et al. 1995; Mackie et al. 2013). All children underwent genetic testing including testing for 22q11 deletion.

\section{Outcome assessment}

As previously described, children in the cohort were assessed at 6-10 months and at 18-24 months of age by multidisciplinary teams at one of 5 centers participating in the Western Canadian Complex Pediatric Therapies
Follow-up Group: Calgary and Edmonton, Alberta; Regina and Saskatoon, Saskatchewan; and Winnipeg, Manitoba (Robertson et al. 2011). At the 18-24 month follow up visit children were assessed using several psychological tools, had a clinical interview and physical examination by a pediatrician experienced in developmental follow up, and completed standardized parentalreport questionnaires (history of hospitalization, family socioeconomic status) as previously described (Neufeld et al. 2008). Hearing was evaluated by certified audiologists. The Bayley Scales of Infant \& Toddler Development-3rd Edition (Bayley-III), a measure of early child development, was administered by experienced pediatric psychologists or psychometrists under the supervision of a psychologist (Bayley 2006). The Bayley-III yields Cognitive, Motor and Language Composite Scores each expressed as a standard score with a mean of 100 and standard deviation (SD) of 15. Subscales include Receptive and Expressive Communication and Fine and Gross Motor with a mean of 10 and a SD of 3.

\section{Statistical analysis}

All statistical analyses were performed with Intercooled STATA Version 12.0 (College Station, Texas) (2011). All tests were two-sided (where applicable) and significance was defined as $p$ value $<0.05$. A correction for multiple comparisons was not applied given the exploratory nature of this work. Bivariate analyses, $X^{2}$ analyses or Fisher's exact test where appropriate, were used to compare Cognitive, Motor and Language Scores of the Bayley-III by presence or absence of VSD and perinatal and maternal characteristics. Continuous variables were compared between groups using Student's t test (two-sided). Descriptive statistics and bivariate analysis were used to identify differences based on the presence or absence of a VSD. Cognitive, Motor and Language Composite Scores were compared to those of the Bayley-III normative sample where $2.27 \%$ of the sample had a score $<70$ and $<85$ (15.86 \%) (Bayley 2006).

Multivariate linear and logistic regression models were developed with a dependent variable of the Language Composite of the Bayley-III. In addition, models were also developed with dependent variables of Receptive Communication or Expressive Communication scores less than 7. Independent variables were included in the models if they were significantly associated with the outcome at $\mathrm{p}<0.2$ in preliminary univariate analysis and then removed in a backwards stepwise fashion to yield a parsimonious model. Multicolinearity was screened for prior to inclusion in multivariate models. Adjusted odds ratios (for language delay), effect sizes (for Language Composite Score) and $95 \%$ confidence intervals are reported. 
Research ethics board approval was obtained from each site, and all parents or guardians provided written informed consent. Funding agencies had no role in data interpretation.

\section{Results}

\section{Description of cohort}

A flowchart of recruitment participation and loss to follow up is presented in Fig. 1. A total of 103 children underwent ASO during the time period. One child was excluded from the cohort as the child had an identified genetic syndrome and one child who underwent ASO died at day 106 of life of complications of preterm birth. Three children were not eligible for inclusion in this analysis as they were assessed with the Bayley Scales of Infant Development-2nd Edition. Of those eligible for inclusion, 2 children were lost to follow-up and 5 children still had results pending at time of the analysis. In this study 91 of 98 eligible children (93\%) had follow up outcomes with Bayley-III and were included in this analysis. There were no differences in demographic variables between those who were included in the analysis and those who were excluded.

\section{Perioperative and demographic characteristics}

Demographic and perioperative characteristics of neonates are presented in Tables 1 and 2. The majority of children did not have a VSD $(n=60)$. Of those a lower

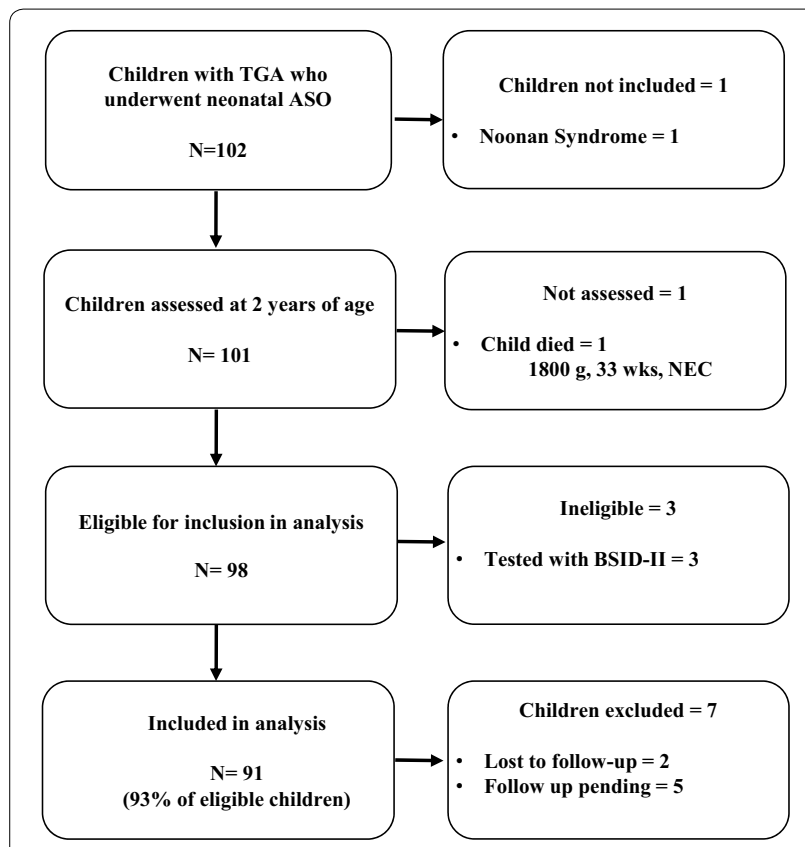

Fig. 1 Study flowchart of recruitment of participants between July 2004 and June 2010 for neonates who underwent neonatal arterial switch operation (ASO) for transposition of the great arteries (TGA) proportion were outborn (52 vs. $73 \%, \mathrm{p}=0.038$ ) and had a pre-operative BAS ( 58 vs. $80 \%, \mathrm{p}=0.026$ ). Children with VSD had a longer cross clamp time (73.9 vs. $62.7 \mathrm{~min}, \mathrm{p}=0.019)$, a higher post-operative lactate on day 1 ( 4.8 vs $3.9 \mathrm{mmol} / \mathrm{L}, \mathrm{p}=0.010)$ and were more likely to require dialysis or have a creatinine $>100 \mu \mathrm{mol} / \mathrm{L}$ (23 vs. $7 \%, \mathrm{p}=0.041)$. No children in this cohort received extracorporeal membrane oxygenation, cardiopulmonary resuscitation, ventricular-assist device or heart transplant.

Brain imaging, either ultrasound or magnetic resonance imaging, were not adequate for inclusion in this analysis as these were not consistently obtained perioperatively. In addition, information on seizures could not be rigorously considered here because electroencephalograms (EEGs) were not consistently obtained.

\section{Neurocognitive outcomes}

There were no differences in cognitive, motor and language outcomes based on presence of VSD (Tables 3, 4 ). Only $3 \%$ of children had a Cognitive score $<70$ (2SD below the mean) while $11 \%$ had a Language score $<70$ and $4 \%$ had a Motor score $<70.12 \%$ of children had Cognitive and Motor scores $<85$ (1SD below the mean). Compared to the normative sample for the Bayley-III, children with TGA were 4.7 times more likely to have a Language score $<70$ and 1.8 times more likely to have a Language score $<85$ (Fig. 2).

\section{Language outcomes}

A parsimonious logistic regression model was developed with an outcome of Language Composite $<85$ ) on the Bayley-III and adjusted odds ratios (AOR) for patient and perioperative characteristics for children are presented in Table 5. The final model has an overall pseudo $R^{2}$ of 0.32 . Maternal education less than 12 years (AOR 19.3, $95 \%$ CI 2.5-148.0), cross-clamp time (AOR 14.5, $95 \%$ CI 3.1-68.5), DHCA (AOR 5.8, $95 \%$ CI 1.3-26.7), and days of ventilation (AOR 1.2, $95 \%$ CI 1.0-1.3) were risk factors for language delay. Female sex, ventricular septal defect and birth at the surgical center were controlled for in the model although not significant predictors of delay. Separate multiple logistic regression models were also developed with receptive or expressive Language scores less than 7 as the outcome of interest. Both models had maternal education less than 12 years as the main predictor of delay (data not shown).

A parsimonious linear regression model was generated with an outcome of language delay (Language Composite Score $<85$ ). The model included maternal education and cross clamp time and had an overall $\mathrm{R}^{2}$ of 0.203 . The language constant was 97.6 (95 \% CI 93.2-101.9) and children who had mothers with less than a Grade 
Table 1 Preoperative and demographic characteristics of neonates requiring arterial switch operation for transposition of the great arteries in the period 2004-2010

\begin{tabular}{|c|c|c|c|c|}
\hline \multirow[t]{2}{*}{ Variables } & \multirow{2}{*}{$\begin{array}{l}\text { Total } \\
\mathrm{N}=91 \\
\mathrm{n}(\%)\end{array}$} & \multicolumn{2}{|c|}{ Ventricular septal defect } & \multirow[t]{2}{*}{$p$ value } \\
\hline & & $\begin{array}{l}\text { No VSD } \\
N=60 \\
n(\%)\end{array}$ & $\begin{array}{l}\text { VSD } \\
N=31 \\
n(\%)\end{array}$ & \\
\hline \multicolumn{5}{|l|}{ Demographic and birth characteristics } \\
\hline Male & $61(67 \%)$ & $38(63 \%)$ & $23(74 \%)$ & 0.30 \\
\hline Antenatal diagnosis: yes & $27(30 \%)$ & $17(28 \%)$ & $10(32 \%)$ & 0.70 \\
\hline Birth gestation (weeks) & $39.0(1.8)$ & $39.1(1.9)$ & $38.8(1.5)$ & 0.60 \\
\hline Birth weight (g) & $3367.6(569)$ & $3370.1(597)$ & $3363.0(517)$ & 0.96 \\
\hline Apgar at 5 min & $8.1(1.2)$ & $7.9(1.3)$ & $8.3(0.9)$ & 0.24 \\
\hline Socioeconomic index (Blishen) & $46.3(13.9)$ & $45.6(12.9)$ & $47.7(15.8)$ & 0.50 \\
\hline Total years of mother's schooling & $14.1(3.3)$ & $13.6(2.9)$ & $15.1(3.9)$ & 0.06 \\
\hline \multicolumn{5}{|l|}{ Guardianship } \\
\hline Parents & $81(89 \%)$ & $52(87 \%)$ & $29(94 \%)$ & \\
\hline Mother & $6(7 \%)$ & $4(7 \%)$ & $2(6 \%)$ & \\
\hline Father & $1(1 \%)$ & $1(2 \%)$ & $0(0 \%)$ & \\
\hline Other & $3(3 \%)$ & $3(5.0 \%)$ & $0(0 \%)$ & 1.00 \\
\hline \multicolumn{5}{|l|}{ Race } \\
\hline European/white & $70(84 \%)$ & $45(80 \%)$ & $25(93 \%)$ & \\
\hline Original peoples & $2(2 \%)$ & $2(4 \%)$ & $0(0 \%)$ & \\
\hline Other & $11(13 \%)$ & $9(16 \%)$ & $2(7 \%)$ & 0.32 \\
\hline Delivery at hospital outside of the region of the operating site & $60(66 \%)$ & $44(73 \%)$ & $16(52 \%)$ & 0.04 \\
\hline \multicolumn{5}{|l|}{ Preoperative characteristics } \\
\hline Pre-operative septostomy & $66(73 \%)$ & $48(80 \%)$ & $18(58 \%)$ & 0.03 \\
\hline Modified inotrope score & $7.7(9.7)$ & $7.5(9.8)$ & $8.1(9.6)$ & 0.78 \\
\hline Plasma lactate (mmol/L) & $3.4(3.1)$ & $3.3(3.3)$ & $3.6(2.7)$ & 0.65 \\
\hline Days ventilated (days) & $5.3(4.7)$ & $5.2(3.1)$ & $5.5(6.9)$ & 0.85 \\
\hline Highest serum creatinine $(\mu \mathrm{mol} / \mathrm{L})$ & $62.6(19.1)$ & $62.2(17.1)$ & $63.3(22.8)$ & 0.80 \\
\hline Sepsis (blood culture confirmed) & $3(3 \%)$ & $3(5 \%)$ & 0 & 0.55 \\
\hline Hydrocortisone cumulative exposure (mg/kg) & $4.2(12.8)$ & $4.8(14.8)$ & $3.1(7.7)$ & 0.49 \\
\hline
\end{tabular}

12 education had Language Composite Scores that were 20.2 points lower $(95 \% \mathrm{CI}-32.4$ to -9.1$)$. Children with a cross clamp time of $70 \mathrm{~min}$ or greater had a Language Composite Score 8.0 points lower $(95 \%$ CI -15.0 to -1.1). Separate multiple linear regression models were also developed with receptive or expressive language as the continuous outcome of interest. Both of the parsimonious models constructed had maternal education less than 12 years as the main predictor of delay (data not shown).

\section{Discussion}

As previously described in recent cohorts, children with TGA who undergo ASO have a low mortality rate as was found in this cohort (Bellinger et al. 1995, 1999; Losay et al. 2001; Bellinger et al. 2003; Neufeld et al. 2008). In addition, as previously noted the children at our center have good preschool neurocognitive, motor and overall health outcomes which are in keeping with the published literature (Bellinger et al. 1995, 1999; Dunbar-Masterson et al. 2001; Hövels-Gürich et al. 2002; Bellinger et al. 2003; Karl et al. 2004; Freed et al. 2006; Creighton et al. 2007; Neufeld et al. 2008). Only $2 \%$ of children had Cognitive scores $<70$ and only 1 child had a Motor score $<70$, scores below 70 would be concerning for the later development of cognitive or motor disabilities. Consistent with cohorts at several centers and over several surgical eras approximately $25 \%$ of children in this study had language delay without evidence of co-occurring delay in cognitive abilities (Bellinger et al. 1995, 1999; HövelsGürich et al. 2002; Karl et al. 2004; Neufeld et al. 2008). The children in this cohort were delayed in comparison to the normative sample from the BSID-III in expressive and receptive communication. Of note, the BSID-III normative sample included children who per born preterm and children with disabilities. In a previous cohort at this 
Table 2 Perioperative characteristics of neonates who required arterial switch operation for transposition of the great arteries in the period 2004 to 2010

\begin{tabular}{|c|c|c|c|c|}
\hline \multirow[t]{2}{*}{ Variables } & \multirow{2}{*}{$\begin{array}{l}\text { Total } \\
\mathrm{N}=91 \\
\mathrm{n}(\%)\end{array}$} & \multicolumn{2}{|c|}{ Ventricular septal defect } & \multirow[t]{2}{*}{$\mathrm{p}$ value } \\
\hline & & $\begin{array}{l}\text { No VSD } \\
\mathrm{N}=60 \\
\mathrm{n}(\%)\end{array}$ & $\begin{array}{l}\text { VSD } \\
\mathrm{N}=31 \\
\mathrm{n}(\%)\end{array}$ & \\
\hline \multicolumn{5}{|l|}{ Operative } \\
\hline Year at surgery & $2007.3(2.2)$ & $2007.3(1.9)$ & $2007.4(2.6)$ & 0.80 \\
\hline Age at surgery (days) & $11.5(14.8)$ & $9.1(3.8)$ & $16.2(24.4)$ & 0.12 \\
\hline Weight at surgery (kg) & $3.485(0.669)$ & $3.445(0.607)$ & $3.562(0.781)$ & 0.43 \\
\hline Cardiopulmonary bypass time (min) & $120.6(39.8)$ & $116.8(32.9)$ & $128.0(50.3)$ & 0.27 \\
\hline Cross-clamp time (min) & $66.5(18.5)$ & $62.7(14.0)$ & $73.9(23.6)$ & 0.02 \\
\hline Use of deep hypothermic circulatory arrest & $60(66 \%)$ & $42(70 \%)$ & $18(58 \%)$ & 0.26 \\
\hline Need for re-cardiopulmonary bypass during first $O R$ & $7(8 \%)$ & $3(5 \%)$ & $4(13 \%)$ & 0.22 \\
\hline \multicolumn{5}{|l|}{ Post-operative, day 1} \\
\hline Modified inotrope score & $8.7(7.2)$ & $8.5(7.5)$ & $9.1(6.8)$ & 0.70 \\
\hline Plasma lactate (mmol/L) & $4.2(1.6)$ & $3.9(1.6)$ & $4.8(1.5)$ & 0.01 \\
\hline Time for lactate to return to 2 or less (h) & $10.9(8.4)$ & $10.3(8.9)$ & $12.0(7.3)$ & 0.36 \\
\hline Highest serum creatinine $(\mu \mathrm{mol} / \mathrm{L})$ & $51.5(12.3)$ & $50.3(11.9)$ & $53.8(13.0)$ & 0.19 \\
\hline \multicolumn{5}{|l|}{ Post-operative, day $2-5$} \\
\hline Modified inotrope score & $7.7(7.7)$ & $7.1(6.7)$ & $8.7(9.3)$ & 0.41 \\
\hline Plasma lactate (mmol/L) & $2.2(1.1)$ & $2.2(1.2)$ & $2.3(0.8)$ & 0.82 \\
\hline Serum creatinine $(\mu \mathrm{mol} / \mathrm{L})$ & $61.5(22.2)$ & $61.5(24.3)$ & $61.3(17.9)$ & 0.97 \\
\hline \multicolumn{5}{|l|}{ Post-operative, day $6+$} \\
\hline Modified inotrope score & $1.2(2.6)$ & $1.2(2.6)$ & $1.4(2.6)$ & 0.74 \\
\hline Plasma lactate (mmol/L) & $1.1(0.4)$ & $1.1(0.35)$ & $1.2(0.49)$ & 0.21 \\
\hline Serum creatinine $(\mu \mathrm{mol} / \mathrm{L})$ & $47.0(20.7)$ & $45.2(20.9)$ & $50.4(20.3)$ & 0.26 \\
\hline \multicolumn{5}{|l|}{ All post-operative time } \\
\hline Ventilation (days) & $6.2(3.5)$ & $6.0(3.5)$ & $6.7(3.4)$ & 0.38 \\
\hline Admission to PICU (days) & $10.8(5.0)$ & $10.5(4.9)$ & $11.4(5.2)$ & 0.37 \\
\hline \multicolumn{5}{|l|}{ Overall/any time } \\
\hline All hospital days ( $\mathrm{SCH})$ & $19.1(8.4)$ & $18.1(8.3)$ & $21.0(8.4)$ & 0.12 \\
\hline Convulsions & $6(7 \%)$ & $2(4 \%)$ & $3(14 \%)$ & 0.41 \\
\hline Ventilation (days) & $11.0(5.5)$ & $11.1(5.4)$ & $10.7(5.7)$ & 0.73 \\
\hline Sepsis pre or post-operative (blood culture positive) & $7(8 \%)$ & $3(6 \%)$ & $3(10 \%)$ & 0.42 \\
\hline Dialysis & $4(4 \%)$ & $1(2 \%)$ & $3(10 \%)$ & 0.11 \\
\hline Dialysis or creatinine $>100(\mu \mathrm{mol} / \mathrm{L})$ & $11(12 \%)$ & $4(7 \%)$ & $7(23 \%)$ & 0.04 \\
\hline
\end{tabular}

center for children undergoing surgery for TGA 1996$2004,26 \%$ had speech or language difficulties at 5 years of age (Neufeld et al. 2008). Children with early language delay are at risk in adolescence and adulthood of poor developmental and mental health outcomes including communication, cognitive, behavioral, social, academic and mental health difficulties (Bashir and Scavuzzo 1992; Johnson et al. 1999; Beitchman et al. 2001; Young et al. 2002; Durkin and Conti-Ramsden 2007). Multiple regression analysis identified maternal education, cross clamp time, requirement for DHCA and total days of ventilation as predictors of language delay. However, only a third of the variance in Language scores was explained by the variables in our model. Multiple regression models with receptive or expressive Language delay as the dependent variable yielded similar parsimonious models. A previous study at our center found maternal education was an important predictor of neurocognitive outcome but variables in regression models only explained roughly $30 \%$ of the variance in outcome (Neufeld et al. 2008). Only 9 mothers in the current study had education less than 12 years so this appears to represent a small subset with an important risk factor for language delay that does not explain the delays seen in the majority of patients. The 
Table 3 Mean scores on the Bayley Scales of Infant \& Toddler Development-3rd Edition after arterial switch operation for transposition of the great arteries for children with and without ventricular septal defect $(n=91)$

\begin{tabular}{|c|c|c|c|c|}
\hline \multirow[t]{2}{*}{ Variable } & \multirow{2}{*}{$\begin{array}{l}\text { Total } \\
\mathrm{n}=91 \\
\text { mean (SD) }\end{array}$} & \multicolumn{2}{|c|}{$\begin{array}{l}\text { Ventricular septal } \\
\text { defect }\end{array}$} & \multirow[t]{2}{*}{ p value } \\
\hline & & $\begin{array}{l}\text { No VSD } \\
n=60 \\
\text { mean (SD) }\end{array}$ & $\begin{array}{l}\text { VSD } \\
\mathrm{n}=31 \\
\text { mean (SD) }\end{array}$ & \\
\hline \multicolumn{5}{|l|}{ Bayley-III } \\
\hline Cognitive composite & $96.9(12.7)$ & $98.4(13.1)$ & $93.8(10.5)$ & 0.10 \\
\hline Language composite & $92.6(17.0)$ & $92.3(18.6)$ & $93.2(13)$. & 0.81 \\
\hline Motor composite & $99.0(12.8)$ & $98.9(13.7)$ & $99.3(11.2)$ & 0.89 \\
\hline \multicolumn{5}{|l|}{ Subscales } \\
\hline $\begin{array}{l}\text { Receptive communi- } \\
\text { cation }\end{array}$ & $9.1(3.0)$ & $9.2(3.3)$ & $8.8(2.4)$ & 0.52 \\
\hline $\begin{array}{l}\text { Expressive communi- } \\
\text { cation }\end{array}$ & $8.4(3.2)$ & $8.2(3.3)$ & $8.8(2.8)$ & 0.40 \\
\hline Fine motor & $10.8(2.7)$ & $10.7(2.7)$ & $11.1(2.7)$ & 0.57 \\
\hline Gross motor & $9.0(2.1)$ & $9.0(2.3)$ & $8.9(1.9)$ & 0.81 \\
\hline
\end{tabular}

Table 4 Development outcomes at 2 years of age as determined by the Bayley Scales of Infant \& Toddler Development-3rd Edition after arterial switch operation for transposition of the great arteries $(n=91)$

\begin{tabular}{lllll}
\hline Outcome & Total & VSD & & $\begin{array}{l}\text { Fisher's } \\
\text { exact, } p\end{array}$ \\
\cline { 3 - 4 } & $n=91$ & No VSD & VSD & \\
& & $n=60$ & $n=31$ &
\end{tabular}

Cognitive

$\begin{array}{lccll}<70 & 2(2.2 \%) & 1(1.7 \%) & 1(3.2 \%) & 1.00 \\ <85 & 10(11.0 \%) & 6(10.0 \%) & 4(12.9) \% & 0.73 \\ \text { Language } & & & & \\ <70 & 9(9.9 \%) & 8(13.3 \%) & 1(3.2 \%) & 0.16 \\ <85 & 26(28.6 \%) & 19(31.7 \%) & 7(22.6 \%) & 0.46\end{array}$

Motor
$<70$

$$
<85
$$

Cognitive $<7$

Receptive communication $<7$

Expressive communication $<7$

Fine motor $<7$

Gross motor $<7$

$\begin{array}{ccll}1(1.1 \%) & 1(1.7 \%) & 0(0 \%) & 1.00 \\ 12(13.2 \%) & 9(15.0 \%) & 3(9.7 \%) & 0.74 \\ 9(9.9 \%) & 6(10.0 \%) & 3(9.7 \%) & 1.00 \\ 22(24.2 \%) & 17(28.3 \%) & 5(16.1 \%) & 0.30 \\ & & & \\ 25(27.5 \%) & 18(30.0 \%) & 7(22.6 \%) & 0.62 \\ 4(4.4 \%) & 4(6.7 \%) & 0(0 \%) & 0.30 \\ 7(7.7 \%) & 5(8.3 \%) & 2(6.5 \%) & 1.00\end{array}$

consistency of language delay across surgical eras and the relatively small amount of variance explained by perioperative variables suggests a considerable influence of currently unidentified risk factors on neurodevelopmental outcome. These risk factors may include the impact of the quality of caregiver-child interaction and parental perception of chronic illness on developmental trajectory.

Deficits often do not become apparent or identifiable until adolescence when demands in social interaction, communication, cognition and executive function increase (Bellinger et al. 1995, 2003; Ovadia et al. 2000; Bellinger and Newburger 2014). Ongoing assessment of neurocognitive outcomes for this cohort is important as a previous surgical era at our center found a higher than expected rate of Autism Spectrum Disorder (ASD) (Neufeld et al. 2008). Approximately $11 \%$ of children had an Intellectual Disability (ID) and $5.5 \%$ had an Autism Spectrum Disorder (Neufeld et al. 2008). Previous studies have also found increased rates of deficits in Executive Function and in symptoms of Attention-Deficit Hyperactivity Disorder (ADHD) (Bellinger et al. 2003). The children in the current cohort were too young for definitive assessment for ID, ASD, ADHD and specific deficits in executive function and this will need to be assessed in the future. A 2012 scientific statement from the American Heart Association which was approved by the American Academy of Pediatrics concluded that "Periodic developmental surveillance, screening, evaluation and re-evaluation throughout childhood may enhance identification of significant deficits, allowing for appropriate therapies and education to enhance later academic, behavioral, psychosocial, and adaptive functioning" (Marino et al. 2012). The results of previous cohorts as well as this cohort highlight the need to continue to assess developmental outcomes for children with TGA (Bellinger et al. 2003; Neufeld et al. 2008; Beca et al. 2009).

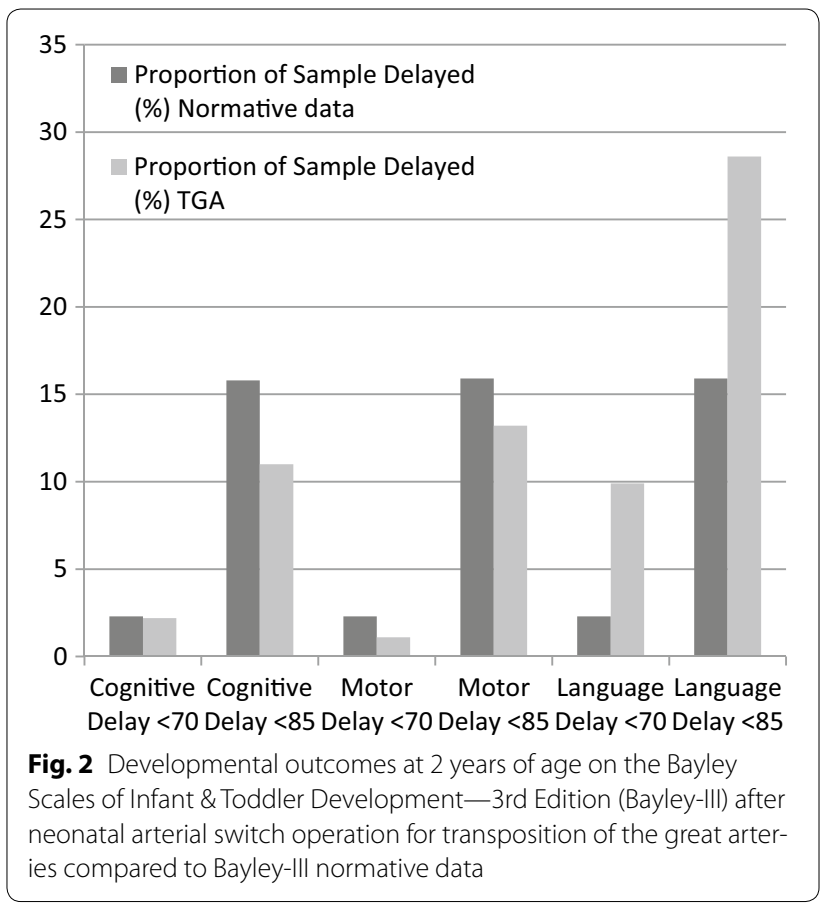


Table 5 Adjusted odds ratios (AOR) for patient and perioperative characteristics for children with language composite $<85$ on the Bayley Scales of Infant \& Toddler Development-3rd Edition at 2 years of age following arterial switch operation for transposition of the great arteries

\begin{tabular}{|c|c|c|c|}
\hline Characteristic & $\begin{array}{l}\text { Delayed } \\
(\mathrm{n}=26) \\
\mathrm{n}(\%)\end{array}$ & $\begin{array}{l}\text { Within normal limits } \\
(n=65) \\
n(\%)\end{array}$ & AOR $(95 \% \mathrm{CI})$ \\
\hline \multicolumn{4}{|l|}{ Patient characteristics } \\
\hline Female sex & $10(38.5)$ & $20(30.8)$ & $2.7(0.7-9.7)$ \\
\hline Maternal education: less than 12 years & $7(26.9)$ & $2(3.1)$ & $19.3(2.5-148.0)$ \\
\hline Ventricular septal defect present & $7(26.9)$ & $24(36.9)$ & $0.4(0.1-1.5)$ \\
\hline Born at surgical center & $4(15.4)$ & $27(41.5)$ & $0.3(0.1-1.3)$ \\
\hline \multicolumn{4}{|l|}{ Perioperative characteristics } \\
\hline Cross-clamp time $\geq 70 \mathrm{~min}$ & $13(50.0)$ & $20(30.8)$ & $14.5(3.1-68.5)$ \\
\hline $\mathrm{DHCA}$ & $19(73.1)$ & $41(63.1)$ & $5.8(1.3-26.7)$ \\
\hline Days of ventilation (for each additional day of ventilation) & $13.6(6.7)$ & $10.0(4.5)$ & $1.2(1.0-1.3)$ \\
\hline
\end{tabular}

A recent meta-analysis found that early interventions in the preterm child can lead to improvements in early and mid-term cognitive outcomes however these benefits were not sustained at school age (Spittle et al. 2012). Children with phonological and expressive vocabulary difficulties may benefit from speech and language intervention, although the most effective model of delivery is not clear (Law et al. 2003; Cirrin et al. 2010; Spittle et al. 2012; Moharir et al. 2014). Children may benefit from focused post-operative early intervention to improve language and developmental outcomes. In particular, children with mothers with education less than 12 years could be considered for targeted intervention.

As previously reported, cross-clamp time is also a predictor of neurocognitive outcome, underscoring the importance of minimizing interruptions in circulation. The mean cross-clamp times compare favorably with those reported in other studies (Bellinger et al. 1995). Cross-clamp time, DHCA and days of ventilation are most likely markers of complexity of surgical intervention and severity of illness. Presence of a VSD was not associated with poorer outcome in this study.

Balloon atrial septostomy was also not a predictor of outcome in this study but was in previous cohorts at our center that considered 5 year outcomes (Neufeld et al. 2008). Neonatal hypoxia and abnormal fetal circulation contribute to brain insult (du Plessis and Volpe 2002). In a recent study the rates of peri-operative brain injury as determined by magnetic resonance imaging (MRI) were similar between infants with TGA, hypoplastic left heart syndrome and pulmonary atresia with no difference in rate of injury by need for BAS (Beca et al. 2009). Overall rates of brain injury appeared to be higher in infants with intact VSD although this did not reach statistical significance (Beca et al. 2009).
The mean age at surgery in this group was 9.1 days in neonates without a VSD and 16.2 for those with a VSD. A recent review of 140 infants with d-TGA who had ASO between 2003 and 2012 at a major surgical center in New York found a significant increase in major morbidity and healthcare costs for every day of delay in surgery beyond day 3 of life (Anderson et al. 2014; Karamlou 2014). The authors concluded that "ASO ought to be performed in preterm and term infants no later than the first week of life". They identified day 3 as the optimal day for surgery with increased risk of major morbidity if surgery was performed day $1-3$ with larger increased risk from day 4 and beyond. In addition, earlier operation could reduce the rate of BAS which was high in this group at $73 \%$. A recent meta-analysis found a BAS rate in TGA of between 20 and $54 \%$ (Doshia et al. 3023). The authors concluded that BAS remains an important option for patients with restrictive interatrial communication who will have a delay in surgery due to transport or lack of access to a surgical team (Doshia et al. 2012). Time to surgery was not a risk factor for language delay in this cohort. Additional prospective studies are required to understand the optimal timing for surgery.

The relatively consistent rates of brain injury regardless of specific type of congenital heart disease in that study would be consistent with a brain injury acquired secondary to hypoxia. Brain imaging, either ultrasound or magnetic resonance imaging, were not included in this analysis as this is not consistently obtained perioperatively. Pre and post-operative MRIs to identify timing of acquisition of white matter injury and potential identification of children at risk for delay could be considered.

Information on clinical and nonclinical seizures could not be rigorously analyzed as EEGs were not consistently 
obtained. Previous studies found that electrographic seizures are common after heart surgery and children with clinical seizures or subclinical electrographic seizures were at increased risk of language delay (Rappaport et al. 1998; Clancy et al. 2003, 2005; Gaynor et al. 2007). However, it is not clear how non-convulsive or nonclinical seizures should be managed in this population (Naim et al. 2015). It may be important to recognize the prevalence and predictors of nonclinical seizure activity in this population and determine if this is a potentially modifiable risk factor for language delay. There has been discussion in the literature of monitoring and potential management strategies for non-convulsive seizures in post-operative cardiac surgery patients, but no randomized controlled trials have been published to date (Naim et al. 2015).

Near-infrared spectroscopy (NIRS) has recently been introduced to the clinical environment to measure cerebral oxygenation but these data are not available for the entire cohort. In addition, data related to clinical management decisions based on NIRS were not available. If NIRS leads to a consistent change in preoperative management with subsequent increase in cerebral oxygenation then there is the potential to improve long term neurodevelopmental outcome. In future studies, it will be important to prospectively collect these data as well as the specific clinical care NIRS protocols and management decisions based on NIRS.

The strengths of this study are the high follow up rate, with $93 \%$ of children who underwent ASO included. The prospectively collected predictive variables using standardized study protocols and the experienced research teams that have worked together since 1996 are also considerable strengths. Limitations of this study are that data on some potential confounding factors are lacking or inconsistently collected including indication for BAS, initial and preoperative saturations, indications for delays in surgery and perioperative cerebral oxygenation through NIRS measurements. In addition, assessment by a speech-language pathologist was not possible at all sites and the children identified with language delay may represent an underestimate of the proportion of children with speech and language difficulties. In future studies, measures other than the Bayley-III Language Composite, administered by speech language pathologists, should be considered to better understand the nature of the language delay. While there were no comparison children, standardized measures with population-normative data were administered by experienced clinicians.

\section{Conclusions}

Following ASO for TGA, children have cognitive and motor skills similar to population norms. Overall outcomes are good, but there continue to be important differences in language development with a risk of language delay at 2 years of age. This may be a marker for more concerning developmental and mental health issues as the child ages. As such, children who undergo neonatal cardiac surgery should receive regular developmental surveillance. Supporting families and children post-operatively with early intervention may help improve language outcomes. There were no differences in pre-operative or demographic variables, nor were there differences in cognitive, language or motor outcomes, based on the presence of a VSD. The rate of BAS was high but this was not a predictor of outcome. While perioperative factors account for some differences seen in language outcomes patient characteristics, particularly maternal education, are the strongest predictors of delay. There may be opportunities for pre and/or post-operative neuro-imaging and perioperative NIRS and EEG monitoring to better understand the timing of brain injury and identify potential therapeutic targets and modifiable risk factors. Additionally, prospective studies are required to understand the optimal timing for surgery.

\begin{abstract}
Abbreviations
ASO: arterial switch operation; BAS: balloon atrial septostomy; Bayley-III: Bayley Scales of Infant \& Toddler Development-3rd Edition; BSID-II: Bayley Scales of Infant Development-2nd Edition; CPB: cardiopulmonary bypass; DHCA: deep hypothermic circulatory arrest; NEC: necrotizing enterocolitis; PICU: pediatric intensive care unit; TGA: transposition of the great arteries; VSD: ventricular septal defect.
\end{abstract}

\section{Authors' contributions}

MSH carried out the analyses, drafted the initial manuscript, reviewed and revised the manuscript, and approved the final manuscript as submitted. RSS supervised MSH in the conduct of this study, reviewed and revised the manuscript, approved the final manuscript as submitted, and is the corresponding author. CMTR conceptualized and designed the study, designed or selected the data collection instruments, and coordinated and supervised data collection at the participating sites, carried out the initial analyses, critically reviewed and revised the manuscript, and approved the final manuscript as submitted. AJ, GA, DC, DBR and IMR: RSS, AJ, GA, DBR and IMR conceptualized and designed the study, designed or selected the data collection instruments, reviewed and revised the manuscript, and approved the final manuscript as submitted. All authors read and approved the final manuscript.

\begin{abstract}
Author details
${ }^{1}$ Department of Pediatrics, University of Alberta, Edmonton, $A B$, Canada. ${ }^{2}$ Department of Pediatrics, University of Calgary, Calgary, AB, Canada. ${ }^{3}$ Pediatric Rehabilitation Outcomes Unit, Glenrose Rehabilitation Hospital, Edmonton, $A B$, Canada. ${ }^{4}$ Department of Surgery, University of Alberta, Edmonton, AB, Canada. ${ }^{5}$ Department of Community Health Sciences, Cumming School of Medicine, University of Calgary, Calgary, AB, Canada.
\end{abstract}

\section{Acknowledgements}

We would like to sincerely thank the children and their families, the NICUs, PICUs and Surgical Teams at the Stollery Children's Hospital and the Western Canadian Complex Pediatric Therapies Follow-up Program including P Blakely, Saskatoon, Saskatchewan and J Bodani, Regina, Saskatchewan.

We thank the families of these children for their active participation in the developmental sites across western Canada and their commitment to this project. We sincerely thank the research and data coordinators and psychologists who made this research study possible: $\mathrm{H}$. Christianson and D. Anseeuw-Deeks, Calgary, Alberta, Canada; K. Penner, J. Bow, and V. Debooy, 
Winnipeg, Manitoba, Canada; B. Acton, Saskatoon, Saskatchewan, Canada; S. Selzer and H. Switzer, Regina, Saskatchewan, Canada; and Jill Tomlinson, W. Biggs, Edmonton, Alberta, Canada.

\section{Competing interests}

The authors have no financial relationships relevant to this article to disclose. The authors have no conflicts of interest to disclose.

\section{Ethics approval and consent to participate}

Research ethics board approval was obtained from each site, and all parents or guardians provided written informed consent. Funding agencies had no role in data interpretation.

\section{Funding}

The Follow-up Program was made possible in part by operating grants from Alberta Health and Wellness (1999-2006), Child Health and the individual follow-up hospital sites.

Received: 2 November 2015 Accepted: 21 September 2016 Published online: 29 September 2016

\section{References}

Anderson BR, Ciarleglio AJ, Hayes DA et al (2014) Earlier arterial switch operation improves outcomes and reduces costs for neonates with transposition of the great arteries. J Am Coll Cardiol 63:481-487. doi:10.1016/j. jacc.2013.08.1645

Bashir AS, Scavuzzo A (1992) Children with language disorders: natural history and academic success. J Learn Disabil 25:53-65. doi:10.1177/002221949202500109

Bayley N (2006) Bayley scales of infant development: technical manual, 3rd edn. The Psychological Corporation, Antonio

Beca J, Gunn J, Coleman L et al (2009) Pre-operative brain injury in newborn infants with transposition of the great arteries occurs at rates similar to other complex congenital heart disease and is not related to balIoon atrial septostomy. J Am Coll Cardiol 53:1807-1811. doi:10.1016/j. jacc.2009.01.061

Beitchman JH, Wilson B, Johnson CJ et al (2001) Fourteen-year followup of speech/language-impaired and control children: psychiatric outcome. J Am Acad Child Adolesc Psychiatry 40:75-82. doi:10.1097/00004583-200101000-00019

Bellinger DC, Newburger JW (2014) Neuropsychological, psychosocial, and quality-of-life outcomes in children and adolescents with congenital heart disease. Prog Pediatr Cardiol 29:87-92. doi:10.1016/j.ppedcard.2010.06.007

Bellinger DC, Jonas RA, Rappaport LA et al (1995) Developmental and neurologic status of children after heart surgery with hypothermic circulatory arrest or low-flow cardiopulmonary bypass. N Engl J Med 332:549-555. doi:10.1056/NEJM199503023320901

Bellinger DC, Wypij D, Kuban KCK et al (1999) Developmental and neurological status of children at 4 years of age after heart surgery with hypothermic circulatory arrest or low-flow cardiopulmonary bypass. Circulation 100:526-532. doi:10.1161/01.CIR.100.5.526

Bellinger DC, Wypij D, duPlessis AJ et al (2003) Neurodevelopmental status at eight years in children with dextro-transposition of the great arteries: the Boston circulatory arrest trial. J Thorac Cardiovasc Surg 126:1385-1396. doi:10.1016/S0022-5223(03)00711-6

Blishen BR, Carroll WK, Moore C (1987) The 1981 socioeconomic index for occupations in Canada. Can Rev Sociol Can Sociol 24:465-488. doi:10.1111/j.1755-618X.1987.tb00639.x

Cirrin FM, Schooling TL, Nelson NW et al (2010) Evidence-based systematic review: effects of different service delivery models on communication outcomes for elementary school-age children. Lang Speech Hear Serv Sch 41:233-264. doi:10.1044/0161-1461(2009/08-0128)

Clancy RR, McGaurn SA, Wernovsky G et al (2003) Risk of seizures in survivors of newborn heart surgery using deep hypothermic circulatory arrest. Pediatrics 111:592-601. doi:10.1542/peds.111.3.592

Clancy RR, Sharif U, Ichord R et al (2005) Electrographic neonatal seizures after infant heart surgery. Epilepsia 46:84-90. doi:10.1111/j.0013-9580.2005.22504.x
Creighton DE, Robertson CMT, Sauve RS et al (2007) Neurocognitive, functional, and health outcomes at 5 years of age for children after complex cardiac surgery at 6 weeks of age or younger. Pediatrics 120:e478-e486. doi:10.1542/peds.2006-3250

Doshia H, Venugopal P, MacArthur K (2012) Does a balloon atrial septostomy performed before surgery increase adverse neurological outcomes? Interact CardioVasc Thorac Surg 15:141-144. doi:10.1093/icvts/ivr145

du Plessis AJ, Volpe JJ (2002) Perinatal brain injury in the preterm and term newborn. Curr Opin Neurol 15:151-157

Dunbar-Masterson C, Wypij D, Bellinger DC et al (2001) General health status of children with D-transposition of the great arteries after the arterial switch operation. Circulation 104:I-138-I-142. doi:10.1161/hc37t1.094782

Durkin K, Conti-Ramsden G (2007) Language, social behavior, and the quality of friendships in adolescents with and without a history of specific language impairment. Child Dev 78:1441-1457. doi:10.1111/j.1467-8624.2007.01076.x

Freed DH, Robertson CMT, Sauve RS et al (2006) Intermediate-term outcomes of the arterial switch operation for transposition of great arteries in neonates: alive but well? J Thorac Cardiovasc Surg 132:845-852. doi:10.1016/j. jtcvs.2006.05.046

Gaynor JW, Wernovsky G, Jarvik GP et al (2007) Patient characteristics are important determinants of neurodevelopmental outcome at one year of age after neonatal and infant cardiac surgery. J Thorac Cardiovasc Surg 133:1344-1353, 1353.e1-3. doi:10.1016/j.jtcvs.2006.10.087

Hövels-Gürich HH, Seghaye M-C, Schnitker R et al (2002) Long-term neurodevelopmental outcomes in school-aged children after neonatal arterial switch operation. J Thorac Cardiovasc Surg 124:448-458. doi:10.1067/ mtc.2002.122307

Johnson CJ, Beitchman JH, Young A et al (1999) Fourteen-year follow-up of children with and without speech/language impairments speech/language stability and outcomes. J Speech Lang Hear Res 42:744-760

Karamlou T (2014) Optimal timing for arterial switch in neonates with transposition of the great arteries: an elusive target. J Am Coll Cardiol 63:488-489. doi:10.1016/j.jacc.2013.09.054

Karl TR, Hall S, Ford G et al (2004) Arterial switch with full-flow cardiopulmonary bypass and limited circulatory arrest: neurodevelopmental outcome. J Thorac Cardiovasc Surg 127:213-222. doi:10.1016/j. jtcvs.2003.06.001

Law J, Garrett Z, Nye C (2003) Speech and language therapy interventions for children with primary speech and language delay or disorder. Cochrane Database Syst Rev. doi:10.1002/14651858.CD004110

Losay J, Touchot A, Serraf A et al (2001) Late outcome after arterial switch operation for transposition of the great arteries. Circulation 104:I-121-|-126. doi:10.1161/hc37t1.094716

Mackie AS, Alton GY, Dinu IA et al (2013) Clinical outcome score predicts the need for neurodevelopmental intervention after infant heart surgery. J Thorac Cardiovasc Surg 145:1248.e2-1254.e2. doi:10.1016/j. jtcvs.2012.04.029

Marino BS, Lipkin PH, Newburger JW et al (2012) Neurodevelopmental outcomes in children with congenital heart disease: evaluation and management: a scientific statement from the American Heart Association. Circulation 126:1143-1172. doi:10.1161/CIR.0b013e318265ee8a

Moharir M, Barnett N, Taras J et al (2014) Speech and language support: how physicians can identify and treat speech and language delays in the office setting. Paediatr Child Health 19:13-18

Naim MY, Gaynor JW, Chen J et al (2015) Subclinical seizures identified by postoperative electroencephalographic monitoring are common after neonatal cardiac surgery. J Thorac Cardiovasc Surg 150:169-180. doi:10.1016/j. jtcvs.2015.03.045

Neufeld RE, Clark BG, Robertson CMT et al (2008) Five-year neurocognitive and health outcomes after the neonatal arterial switch operation. J Thorac Cardiovasc Surg 136:1413-1421, 1421.e1-1421.e2. doi:10.1016/j. jtcvs.2008.05.011

Ovadia R, Hemphill L, Winner K, Bellinger D (2000) Just pretend: participation in symbolic talk by children with histories of early corrective heart surgery. Appl Psycholinguist 21:321-340

Rappaport L, Wypij D, Bellinger DC et al (1998) Relation of seizures after cardiac surgery in early infancy to neurodevelopmental outcome. Circulation 97:773-779. doi:10.1161/01.CIR.97.8.773

Robertson CMT, Sauve RS, Joffe AR et al (2011) The registry and follow-up of complex pediatric therapies program of Western Canada: a mechanism 
for service, audit, and research after life-saving therapies for young children. Cardiol Res Pract 2011:965740. doi:10.4061/2011/965740

Spittle A, Orton J, Anderson P et al (2012) Early developmental intervention programmes post-hospital discharge to prevent motor and cognitive impairments in preterm infants. Cochrane Database Syst Rev 12:CD005495. doi:10.1002/14651858.CD005495.pub3

Stata Statistical Software: Release 12 (2011)

Wernovsky G, Wypij D, Jonas RA et al (1995) Postoperative course and hemodynamic profile after the arterial switch operation in neonates and infants. A comparison of low-flow cardiopulmonary bypass and circulatory arrest. Circulation 92:2226-2235. doi:10.1161/01.cir.92.8.2226
Williams W (2003) Outcomes of 829 neonates with complete transposition of the great arteries 12-17 years after repair. Eur J Cardio-Thorac Surg 24:1-10. doi:10.1016/S1010-7940(03)00264-1

Wypij D, Newburger JW, Rappaport LA et al (2003) The effect of duration of deep hypothermic circulatory arrest in infant heart surgery on late neurodevelopment: the Boston circulatory arrest trial. J Thorac Cardiovasc Surg 126:1397-1403. doi:10.1016/S0022-5223(03)00940-1

Young AR, Beitchman JH, Johnson C et al (2002) Young adult academic outcomes in a longitudinal sample of early identified language impaired and control children. J Child Psychol Psychiatry 43:635-645. doi:10.1111/1469-7610.00052

\section{Submit your manuscript to a SpringerOpen ${ }^{\circ}$ journal and benefit from:}

- Convenient online submission

\section{- Rigorous peer review}

- Immediate publication on acceptance

- Open access: articles freely available online

- High visibility within the field

- Retaining the copyright to your article 